\title{
'PASCUAL APORTA', UN MARCO DE GESTIÓN RESPONSABLE PARA LOS OBJETIVOS DE DESARROLLO SOSTENIBLE
}

\author{
Autor: Joseba Arano \\ joseba.arano@calidadpascual.com \\ Director de Personas, Calidad y Gestión Ética, Responsable y Excelente
}

\begin{abstract}
Resumen
Calidad Pascual es una sociedad de propiedad familiar, constituida en España en 1969 que opera principalmente en el sector de la alimentación y bebidas. Pascual Aporta es el marco donde se engloban todas las iniciativas de gestión responsable de Pascual para dar respuesta a los desafíos marcados en los Objetivos de Desarrollo Sostenible (ODS). Pascual Aporta relaciona la actividad de la empresa con los ODS en los territorios en los que la compañía actúa: Bienestar, Desarrollo y Medioambiente. Este compromiso se traslada a la cadena de valor desde la aplicación de los valores en el día a día: integridad, cercanía, pasión, calidad e innovación. Estas convicciones impulsan la actividad de la compañía, respondiendo a las expectativas de sus grupos de interés y generando valor compartido con toda la sociedad a lo largo de 50 años.
\end{abstract}


Palabras clave: Pascual Aporta; ODS; grupos de interés; valores corporativos; gestión responsable; cadena de valor; bienestar; desarrollo; medioambiente.

'Pascual Aporta' a responsible management framework for sustainable development objectives

\section{Abstract}

Pascual is a family-owned business established in Spain in 1969 that operates primarily in the food and beverage sector through Calidad Pascual and its subsidiary companies. Pascual Aporta is the framework that includes all of Pascual's Responsible Management initiatives to meet Sustainable Development Goals from the UN 2030 Agenda for Sustainable Development. Pascual Aporta links the company's agenda to the Sustainable Development Goals in the areas in which it acts: Well-being, Development and Environment. Committed to contributing to the value chain by applying values in the daily work: integrity, closeness, passion, quality and innovation. These convictions drive the company's activity, in response to their stakeholders' expectations and generating shared value with society at large for 50 years.

Keywords: Pascual Aporta; Sustainable Development Goals; stakeholders; corporate values; responsible management; value chain; well-being; development; environment.

Fecha de recepción: 18/11/2019.

Fecha de aceptación: 20/11/2019.

\section{CALIDAD PASCUAL, EMPRESA FAMILIAR DEL SECTOR ALIMENTARIO}

Calidad Pascual, empresa familiar con un conjunto de valores consolidado y diferenciador, referente en el sector de la alimentación, inició su andadura en 1969 en Aranda de Duero, Burgos, para implantarse en poco tiempo en toda España y exportar hoy a 67 países. Con una gama de más de 200 productos, 6 plantas, 27 delegaciones a través de su empresa de distribución Qualianza y más de 2.200 empleados, su principal actividad es la preparación, envasado, distribución y venta, entre otros, de lácteos y derivados con la marca Pascual, aguas minerales Bezoya, bebidas vegetales Vivesoy, Bifrutas y Mocay 'Maestros del café'. 
Calidad Pascual complementa su portfolio con otros productos de valor añadido, gracias a alianzas con empresas como Idilia foods (Cola Cao, Nocilla y Okey), Upfield (Flora), Agua das Pedras Salgadas, Kellogg, Heinz, Conservas Garavilla (Isabel), Aceites y Vinagres Cosecha de Borges, La Zaragozana (Ambar y Malen) y AUARA.

Gracias a la actividad de Calidad Pascual, cada vez son más las personas que disfrutan de un nuevo concepto de calidad, más amplio y global, más allá del desarrollo de productos saludables. Calidad Pascual tiene como principio inspirador la aportación de valor y calidad a la vida de las personas, entendida como un compromiso colectivo de superación en la búsqueda diaria de su bienestar.

\section{EL MODELO DE EMPRESA DE CALIDAD PASCUAL Y LA GESTIÓN RESPONSABLE}

\section{MODELO DE EMPRESA: VISIÓN, MISIÓN, VALORES, GOBIERNO Y ESTRATEGIA}

La familia Pascual Gómez-Cuétara es una familia empresaria, una estructura basada en los mismos lazos de sangre, emociones y relaciones que cualquier otra familia, pero que tiene como característica esencial la necesidad de crear actividad empresarial que genere bienestar a las personas y desarrollo social a su alrededor. La misión de la familia empresaria es mejorar la sociedad en la que viven sus miembros.

Cada generación de la familia tiene el objetivo de desarrollar el legado recibido y hacerlo más grande para que la siguiente generación pueda seguir cumpliendo la misión de la familia. En la actualidad la segunda generación está al frente de la empresa familiar y a través de sus estructuras trabaja para formar a la tercera generación.

Las empresas de Pascual deben ser referentes en la sociedad, deben abrir caminos y marcar estándares de excelencia que hagan que sean apreciadas y valoradas por la sociedad y todas las personas con las que se interactúe. El liderazgo de Pascual se basa en valores, en una actividad ética apoyada en los cinco valores de la empresa.

Para hacer realidad este mandato, Calidad Pascual ha desarrollado un modelo de empresa que integra la visión, misión y los valores corporativos, es decir, el propósito y la identidad. A partir de este faro se establece el sistema de gobierno corporativo que impulsa comportamientos que se enmarcan en la ética y la responsabilidad. El objetivo es ser referentes dentro de las empresas no cotizadas en la gestión eficiente y excelente de los Órganos de Gobierno Corporativos, 
adecuándose a las recomendaciones de Buen Gobierno contenidas en el Código de Buen Gobierno de las Sociedades Cotizadas, aprobado en febrero de 2015.

Como siguiente eslabón, los órganos de gobierno de la compañía han formulado una serie de Políticas del Consejo de Administración y unos Sistemas de Gestión que marcan la pauta para alcanzar un comportamiento correcto y homogéneo. Con el fin de asegurar el crecimiento sostenible de la compañía, en el medio y en el largo plazo, las Políticas del Consejo de Administración de Pascual velan por que la toma de decisiones estratégicas se realice siempre en el marco de los más elevados estándares de calidad, responsabilidad de integridad, acorde con los valores corporativos. Estas Políticas fomentan la seguridad económica y jurídica. Junto con las políticas, Calidad Pascual ha desarrollado un modelo de gestión propio denominado Mare Nostrum, basado en el ciclo de mejora continua y ampliado con otros elementos de gestión claves para Pascual que permite desarrollar un estilo de trabajo con orientación a la excelencia.

Una vez identificado el propósito y la forma de gobierno corporativo, y ya dotados de los referentes normativos y de gestión básicos, los gestores elaboran la estrategia de negocio tras una escucha exhaustiva a los grupos de interés de la compañía: consumidores, clientes, empleados, proveedores, sociedad y familia propietaria. El impacto de la actividad en el entorno repercute en una serie de actores que tienen una influencia directa en el desarrollo de la empresa. Ellos son su prioridad en la toma de decisiones.

El grupo de interés es por tanto el elemento central del sistema. El punto de referencia de lo que la compañía quiere ser, hacer y decir para cumplir su estrategia y modelo de compañía. Calidad Pascual ha desarrollado un modelo proactivo en la escucha sistemática de sus necesidades y expectativas con diferentes cauces y herramientas de comunicación, participación y diálogo, que culminan en la toma de decisiones de la compañía.

A través de la identificación de los grupos de interés y su sistema de escucha Pascual logra establecer canales de diálogo que permiten conocer sus percepciones, riesgos y oportunidades, enfocando las estrategias con la vista puesta en sus necesidades.

Por último, la empresa identifica aquellos temas más relevantes para sus grupos de interés con el fin de integración en la estrategia y planes de la compañía.

Por tanto, el diálogo con los grupos de interés se construye, por la base, desde la escucha activa de sus expectativas y necesidades, para conectar, en la cúspide, con la estrategia de negocio y gestión de la compañía. 


\section{LA GESTIÓN RESPONSABLE Y SU VINCULACIÓN CON LOS OBJETIVOS DE DESARROLLO SOSTENIBLE}

Hasta aquí una breve descripción del modelo de empresa: propósito, gobierno y estrategia de negocio. Si se analiza específicamente el posicionamiento de Pascual en materia de RSC, es necesaria una doble aproximación.

Desde la perspectiva del gobierno corporativo se observan otros dos aspectos prioritarios. Por un lado, se ha aprobado por el Consejo (una de las cuatro Políticas del Consejo) una Política de Responsabilidad Social Corporativa que simplifica y aglutina la normativa interna sobre esta materia, facilita el control y la supervisión del Consejo de Administración y pasa a ser accionable en planes y proyectos concretos.

Por otro, se potencia la Comisión de RSC, órgano delegado del Consejo de Administración, como impulsor de la política de RSC. Este nuevo órgano juega un rol activo de velar por el cumplimiento de la política de RSC, tanto en el seguimiento del impacto social de la compañía como en asegurar el despliegue de un modelo de gestión responsable en la forma de hacer negocio y llevar a cabo su actividad, siguiendo los estándares del Código de Gobierno Corporativo de Sociedades Cotizadas; aunque Pascual no sea una compañía que cotice en bolsa.

Por el respeto por el medio ambiente, los derechos humanos y laborales y la lucha contra la corrupción, así como por el crecimiento de la mano de las comunidades donde la compañía está presente, Pascual manifiesta un sólido compromiso con los 10 Principios del Pacto Mundial de las Naciones Unidas, así como con los 17 ODS, compromiso necesario y ético para desarrollar una sociedad justa y sostenible.

Por ello, dentro de las atribuciones de la Comisión de Responsabilidad Social Corporativa se encuentra el seguimiento y la supervisión de la vinculación de la compañía con los ODS. Calidad Pascual ha asumido una posición de firme compromiso y acción con los 17 Objetivos de Desarrollo Sostenible (ODS), aprobados en la cumbre de Desarrollo Sostenible de Naciones Unidas de 2015.

En cuanto a la gestión de la RSC propiamente dicha, Calidad Pascual ha creado la dirección de Gestión Ética, Responsable y Excelente para potenciar y desplegar el modelo, la nueva política de RSC del Consejo y su manifestación y plasmación a través del programa Pascual Aporta en todas las sociedades y negocios de la compañía. La verdadera manera de convertir en ventaja competitiva el modelo de gestión es conseguir su integración transversal. A esta aproximación la han denominado enfoque "maceta". 


\section{Enfoque de la gestión responsable en Calidad Pascual}

Modelo previo de gestión responsable en Calidad Pascual

- La RSC era una unidad corporativa central con capacidad ejecutiva y que agrupaba las funciones sobre el impacto social y los intangible.

- La unidad de RSC definía los planes objetivos y mensajes se definen previamente por la unidad centralizada.

- Las Unidades de Negocio eran ejecutoras de los planes, pero no propietarias.

- La Unidad de RSC era el área Accountable.

Nuevo enfoque de gestión responsable en Calidad Pascual

- Creación de una plataforma común en materia de RSC, a través de políticas corporativas y criterios generales

- Las áreas se benefician de la plataforma común y asumen su propia responsabilidad

- El gestor de RSC es un área de soporte y un facilitador para apoyar a las unidades, poner en valor los planes de las áreas y asegurar el cumplimiento de los criterios generales.

- La comisión de RSC del Consejo se convierte en el órgano favorecer la coordinación, la transversalidad y dotar el Consejo de herramientas para asegurar el control.

El gestor de RSC deja de ser una unidad corporativa central con capacidad ejecutiva para convertirse en un área de soporte y un facilitador para apoyar a las unidades a poner en valor los planes de las áreas y asegurar el cumplimiento de los criterios generales. El objetivo es, por tanto, que el negocio y todas las personas que lo integran se gestionen según un modelo de gestión ética, responsable y excelente.

No se persigue un departamento de RSC como órgano staff aparte del negocio. Sino que se produce un retorno al modelo "maceta", a cultivar el terreno apropiado y fertilizarlo para que los principios de gestión ética, responsable y excelente se incorporen en la forma de hacer negocio desde la alta dirección, pasando por los mandos intermedios hasta llegar a toda la organización.

En este planteamiento, el seguimiento de los Objetivos de Desarrollo Sostenible (ODS) sigue siendo una prioridad en la gestión. El modelo "maceta" se extiende entre todas las áreas bajo el paraguas de los ODS. En este sentido, la dirección de Gestión Ética, Responsable y Excelente promueve dentro y fuera de la compañía acciones de difusión, sensibilización y comunicación relacionados con los ODS. 


\section{PROGRAMA PASCUAL APORTA: UN MARCO DE GESTIÓN RESPONSABLE PARA CONTRIBUIR A LOS OBJETIVOS DE DESARROLLO SOSTENIBLE}

Como resumen de lo hasta ahora expuesto cabe señalar que Calidad Pascual persigue un modelo de negocio sostenible en el largo plazo que trabaje para la satisfacción de las expectativas de los grupos de interés gestionando de manera integral los impactos que como compañía genera. En este contexto nace el programa Pascual Aporta. Fruto de la escucha de los grupos de interés, su posterior análisis, los temas materiales derivados del mismo y como respuesta a sus intereses e inquietudes.

Pascual Aporta responde a otro gran reto adicional y complementario. Efectivamente, este sistema de gestión responsable ya inspirado por la naturaleza del negocio en su compromiso con la sostenibilidad y con la escucha de sus grupos de interés, comparte además los temas vertebradores de la agenda post 2015. Por ello, en el proceso de diseño de Pascual Aporta la compañía cruzó los 17 Objetivos y sus 169 metas con los planes y acciones recogidos en el nuevo programa.

Pascual Aporta es una iniciativa puesta en marcha por Calidad Pascual y la Fundación Tomás Pascual y Pilar Gómez-Cuétara con el fin de integrar la gestión responsable en el negocio dando respuesta a los desafíos marcados en los Objetivos de Desarrollo Sostenible (ODS). Este marco de la compañía y todas sus marcas nace con el objetivo de proyectar una imagen coherente y conjunta de las diferentes iniciativas llevadas a cabo en los territorios en los que actúa Pascual: Bienestar, Desarrollo y Medioambiente.

El esfuerzo y dedicación en el desarrollo y el bienestar de la sociedad cristaliza en un marco que engloba las iniciativas, contribuciones y compromisos de gestión responsable relacionados con el Bienestar, Desarrollo y Medioambiente. Unificando, racionalizando y simplificando los proyectos lanzados por las diferentes áreas, permitiendo preservar los ámbitos propios de gestión y, al mismo tiempo, conectarlos de forma estratégica.

Cada territorio engloba unas acciones específicas y cuenta con unos objetivos diferenciados marcados por la compañía. Así, el apartado Bienestar busca contribuir a la salud, bienestar y nutrición de sus grupos de interés. Por su parte Desarrollo, apuesta por ser el mejor lugar para trabajar con empleo de calidad, impulsar una cadena de valor responsable y el impacto positivo en las comunidades. Y, en medioambiente, el objetivo propuesto por la organización es minimizar el impacto medioambiental de su actividad y favorecer la economía circular. 


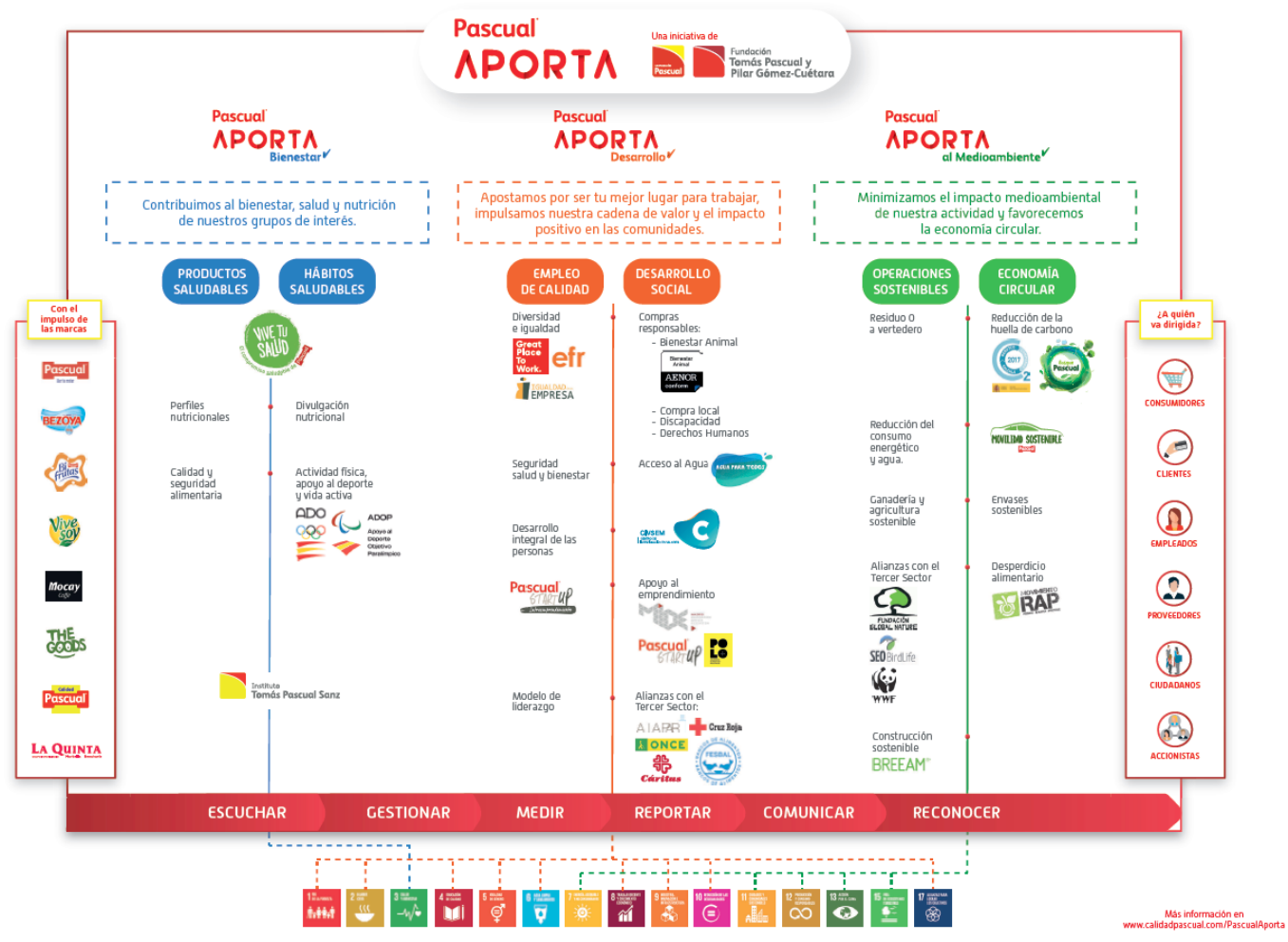

\section{VINCULACIÓN DEL PROGRAMA PASCUAL APORTA CON LOS OBJETIVOS DE DESARROLLO SOSTENIBLE}

Pascual Aporta suma a su plasmación del mandato de la gestión ética, responsable y excelente y del diálogo con los grupos de interés de Calidad Pascual, el establecimiento de conexiones con las metas establecidas por Naciones Unidas en los ODS. De esta forma, Pascual se compromete a continuar contribuyendo a la consecución de los ODS de la Agenda 2030 de Naciones Unidas para poner fin a la pobreza, proteger el planeta y garantizar el bienestar global de las personas, en las que impacta a través de su actividad productiva.

De este primer acercamiento se concluye que Pascual contribuye de forma directa y real, mediante la ejecución del programa Pascual Aporta a 15 de los 17 ODS, incidiendo directamente en 24 de sus metas.

Pascual Aporta Bienestar contribuye al bienestar de los diferentes grupos de interés, tanto con la oferta de productos saludables, como con la promoción de 
hábitos saludables para niños y adultos. Este eje tiene relación con el ODS 3 que persigue promover la salud mental y el bienestar.

Así, la compañía busca impulsar el bienestar integral en las principales facetas de la persona: emocional (valores y empoderamiento), física (nutrición y alimentación) y profesional (desarrollo y empleabilidad) a través de programas como 'Vive tu salud', que expresa y sistematiza el compromiso de la compañía para ayudar a mejorar la salud de la sociedad. O con la labor del Instituto Tomás Pascual Sanz de formación y divulgación relacionadas con la alimentación, nutrición y bienestar de las personas para ayudar a que los ciudadanos y los consumidores desarrollen hábitos de vida más saludables.

Además, respondiendo al desafío de promover la transparencia en la información nutricional en el etiquetado, Pascual es una de las empresas que han firmado el Plan de Colaboración para la Mejora de la Composición de los Alimentos y Bebidas y, desde 2004, está adherida a la Estrategia para la Nutrición, Actividad Física y Prevención de la Obesidad (NAOS) del Ministerio de Sanidad, Consumo y Bienestar Social. La compañía trabaja asimismo en el estudio y aplicación de perfiles nutricionales óptimos para garantizar la adecuación nutricional de los productos de su portfolio. También tiene un firme compromiso con el deporte, y la organización patrocina los programas ADO y ADOP.

El eje de Pascual Aporta Desarrollo está vinculado con diversos ODS, como el 1 y el 2 en facetas tales como el impulso de medidas para la contribución social y laboral en el medio rural o la promoción de prácticas de cultivo sostenible. Así, Pascual mantiene un firme compromiso con los agricultores y ganaderos locales, favoreciendo el desarrollo del empleo en el país. Además del empleo directo, la actividad de la compañía genera puestos de trabajo indirecto como consecuencia de la actividad en el territorio nacional. Pascual se ha convertido en el primer fabricante con todas sus granjas certificadas en Bienestar Animal por AENOR bajo los parámetros del Welfare Quality, el más alto estándar europeo para las evaluaciones en granja; y toda la avena utilizada en sus bebidas vegetales Vivesoy provienen de cultivo $100 \%$ local.

Desde el punto de vista laboral, Pascual Aporta Desarrollo también contribuye a los ODS 4, 5 y 8 a través de diversas iniciativas y programas. La gestión del empleo de calidad es uno de los ejes primordiales de la estrategia de la compañía. Existen a disposición de los empleados diferentes herramientas y canales de diálogo que ayudan a definir conjuntamente la mejor estrategia. Calidad Pascual dispone de la Certificación EFR (Empresa Familiarmente Responsable) de la Fundación Más Familia y es reconocida como una de las mejores empresas para trabajar en España según el ranking Great Place to Work. La empresa tiene también un compromiso con la discapacidad, cuenta ya con un $3 \%$ de personas con discapacidad en su plantilla, 
y ha firmado alianzas con entidades referentes como la ONCE y la Fundación A la Par para la compra de bienes y servicios a centros especiales de empleo.

Pascual Aporta Desarrollo también recorre algunos de los objetivos de los ODS 9,10 y 17 . En concreto, contribuye a los fines de impulsar las innovaciones y la tecnología sostenible aplicada al sector agroalimentario y de potenciar la inclusión social, económica y política de todas las personas, en especial de aquellas en situación de vulnerabilidad. Calidad Pascual impulsa la gestión responsable de su cadena de valor desde la compra local, la inclusión de la discapacidad y el respeto a los Derechos Humanos. La compañía mantiene acuerdos con la ONCE, Cáritas, Bancos de Alimentos, Cruz Roja Española y otras entidades sin ánimo de lucro. Destacan otras iniciativas de apoyo al emprendimiento con la participación en programas como Madrid Innovation Driven Ecosystem (MIDE), Pascual Startup y Polo Positivo, en Burgos. Igualmente, dentro de su Fundación desarrolla CIVSEM (Centro de Investigación en Valores), una iniciativa para formar en valores y en herramientas de coaching para el crecimiento personal y profesional.

El alcance de Pascual Aporta Medioambiente cubre todas las etapas de la cadena de valor, girando en torno al aprovechamiento sostenible, la producción eficiente, el ecodiseño en los envases, la movilidad sostenible, la huella ambiental y con la implicación y visibilidad de toda la compañía. Partiendo de este planteamiento se explica la contribución a los ODS de contenido medioambiental 6, 7, 11, $12,13,15$. La meta de sostenibilidad medioambiental reflejada en la estrategia de la compañía despliega cinco objetivos clave con los cuales ya está trabajando: reducción del 20\% de las emisiones de CO2, reducción del 20\% del consumo de agua y electricidad, alcanzar el 100\% de utilización de PET reciclado en envases y cero residuos a vertedero.

Pascual marcó un hito al convertirse en la primera empresa del sector lácteo en inscribir su huella de carbono en el registro de la Oficina España de Cambio Climático del Ministerio; ya utiliza un $98 \%$ de su energía proveniente de fuentes renovables y ha evitado la emisión a la atmósfera de 980 toneladas de CO2 de forma directa y 1.085 toneladas de forma indirecta fruto de su apuesta por la movilidad sostenible. 


\begin{tabular}{|c|c|c|}
\hline & $\begin{array}{l}\text { Retos identificados en el sector } \\
\text { alimentario }\end{array}$ & $\begin{array}{l}\text { Acciones de Pascual Aporta } \\
\text { alineadas con los ODS }\end{array}$ \\
\hline \multicolumn{3}{|c|}{ Pascual Aporta Bienestar } \\
\hline $\begin{array}{l}\text { ODS } 3 . \\
\text { Salud y } \\
\text { bienestar }\end{array}$ & $\begin{array}{l}\text { - Promover la transparencia en la } \\
\text { información nutricional en el eti- } \\
\text { quetado. } \\
\text { - Extender medidas de promoción de } \\
\text { una dieta saludable. }\end{array}$ & $\begin{array}{l}\text { - Vive tu Salud } \\
\text { - Calidad y seguridad alimentaria } \\
\text { - Oferta de productos saludables } \\
\text { - Fomento de hábitos saludables } \\
\text { - Divulgación nutricional } \\
\text { - Instituto Tomás Pascual Sanz } \\
\text { - Salud y bienestar de la sociedad } \\
\text { - Actividades físicas y deportivas } \\
\text { - Bebé a Bordo }\end{array}$ \\
\hline \multicolumn{3}{|c|}{ Pascual Aporta Desarrollo } \\
\hline $\begin{array}{l}\text { ODS } 1 . \\
\text { Fin de la } \\
\text { pobreza }\end{array}$ & $\begin{array}{l}\text { - Impulsar medidas para la contribu- } \\
\text { ción social y laboral en el medio } \\
\text { rural. }\end{array}$ & $\begin{array}{l}\text { - Aprovisionamiento de soja na- } \\
\text { cional de la máxima calidad y } \\
\text { seguridad } \\
\text { - Mocay Academy } \\
\text { - 'Desayuno Solidario' } \\
\text { - Acuerdo de colaboración con } \\
\text { Cáritas } \\
\text { - Acceso al Agua }\end{array}$ \\
\hline $\begin{array}{l}\text { ODS } 2 . \\
\text { Hambre cero }\end{array}$ & $\begin{array}{l}\text { - Promocionar la gestión agraria efi- } \\
\text { ciente de los recursos naturales. } \\
\text { - Promover prácticas de cultivo sos- } \\
\text { tenible. } \\
\text { - Impulsar el derecho a la alimenta- } \\
\text { ción. }\end{array}$ & $\begin{array}{l}\text { - Aprovisionamiento sostenible y } \\
\text { saludable } \\
\text { - Calidad y seguridad alimentaria } \\
\text { - Acceso al agua }\end{array}$ \\
\hline $\begin{array}{l}\text { ODS } 4 . \\
\text { Educación } \\
\text { de calidad }\end{array}$ & $\begin{array}{l}\text { - Formar en sostenibilidad a empleados } \\
\text { - F proveedores. } \\
\text { - Formar en derechos humanos a } \\
\text { empleados y proveedores. }\end{array}$ & $\begin{array}{l}\text { - Mocay Academy } \\
\text { - Calidad en el empleo } \\
\text { - Beneficios sociales y ventajas para } \\
\text { los empleados } \\
\text { - Apoyo a la familia de los empleados }\end{array}$ \\
\hline $\begin{array}{l}\text { ODS } 5 . \\
\text { Igualdad de } \\
\text { género }\end{array}$ & $\begin{array}{l}\text { - Impulsar medidas contra la discri- } \\
\text { minación laboral por género. }\end{array}$ & - Iniciativa EFR \\
\hline $\begin{array}{l}\text { ODS } 6 . \\
\text { Agua limpia y } \\
\text { saneamiento }\end{array}$ & $\begin{array}{l}\text { - Promover la gestión sostenible del } \\
\text { agua. }\end{array}$ & $\begin{array}{l}\text { - Programa de medio ambiente en } \\
\text { granjas proveedoras } \\
\text { - Ciclo del agua en Calidad Pascual } \\
\text { - Modelo de producción eficiente } \\
\text { - Eficiencia ambiental } \\
\text { - Acceso al agua }\end{array}$ \\
\hline
\end{tabular}




\begin{tabular}{|c|c|c|}
\hline & $\begin{array}{c}\text { Retos identificados en el sector } \\
\text { alimentario }\end{array}$ & $\begin{array}{l}\text { Acciones de Pascual Aporta } \\
\text { alineadas con los ODS }\end{array}$ \\
\hline $\begin{array}{l}\text { ODS } 8 . \\
\text { Trabajo } \\
\text { decente y } \\
\text { crecimiento } \\
\text { económico }\end{array}$ & $\begin{array}{l}\text { Promocionar condiciones laborales } \\
\text { adecuadas y dignas para todas las } \\
\text { personas. }\end{array}$ & $\begin{array}{l}\text { - Aprovisionamiento de soja na- } \\
\text { cional de la máxima calidad y } \\
\text { seguridad } \\
\text { - Mocay Academy } \\
\text { - Pascual StartUp }\end{array}$ \\
\hline $\begin{array}{l}\text { ODS } 9 . \\
\text { Industria, } \\
\text { innovación e } \\
\text { infraestructura }\end{array}$ & $\begin{array}{l}\text { - Impulsar las innovaciones y la tec- } \\
\text { nología sostenible aplicada al sector } \\
\text { agroalimentario. }\end{array}$ & $\begin{array}{l}\text { - Presencia en medios y posiciona- } \\
\text { miento online } \\
\text { - Pascual StartUp } \\
\text { - Instituto Tomás Pascual Sanz }\end{array}$ \\
\hline $\begin{array}{l}\text { ODS } 10 . \\
\text { Reducción } \\
\text { de las } \\
\text { desigualdades }\end{array}$ & $\begin{array}{l}\text { - Impulsar la inclusión social de perso- } \\
\text { nas en situación de vulnerabilidad. }\end{array}$ & $\begin{array}{l}\text { - Iniciativa EFR } \\
\text { - Concurso Está en tu Mano }\end{array}$ \\
\hline $\begin{array}{l}\text { ODS } 17 . \\
\text { Alianzas para } \\
\text { lograr los } \\
\text { objetivos }\end{array}$ & $\begin{array}{l}\text { - Impulsar alianzas para desarrollar } \\
\text { proyectos de sostenibilidad. }\end{array}$ & $\begin{array}{l}\text { - Desayunos Pascual } \\
\text { - Alianzas estratégicas } \\
\text { - Presencia en asociaciones }\end{array}$ \\
\hline \multicolumn{3}{|c|}{ Pascual Aporta Medioambiente } \\
\hline $\begin{array}{l}\text { ODS } 7 . \\
\text { Energía } \\
\text { asequible y no } \\
\text { contaminante }\end{array}$ & $\begin{array}{l}\text { - Impulsar prácticas de eficiencia } \\
\text { energética. } \\
\text { - Potenciar el uso de energías reno- } \\
\text { vables. }\end{array}$ & $\begin{array}{l}\text { - Programa de medio ambiente en } \\
\text { granjas proveedoras } \\
\text { - Máximos estándares de calidad y } \\
\text { eficiencia en nuestros procesos } \\
\text { - Modelo de producción eficiente } \\
\text { - Eficiencia ambiental }\end{array}$ \\
\hline $\begin{array}{l}\text { ODS } 11 . \\
\text { Ciudades y } \\
\text { comunidades } \\
\text { sostenibles }\end{array}$ & $\begin{array}{l}\text { - Impulsar la optimización sostenible } \\
\text { del transporte. }\end{array}$ & $\begin{array}{l}\text { - Movilidad sostenible } \\
\text { - 'Acreditación Flota Ecológica' }\end{array}$ \\
\hline $\begin{array}{l}\text { ODS } 12 . \\
\text { Producción } \\
\text { y consumo } \\
\text { responsables }\end{array}$ & $\begin{array}{l}\text { - Fomentar el consumo responsable. } \\
\text { - Promover la gestión sostenible de } \\
\text { residuos, vertidos y de la contami- } \\
\text { nación. } \\
\text { - Reducir el impacto ambiental de los } \\
\text { envases. } \\
\text { - Impulsar medidas para reducir las } \\
\text { pérdidas y el desperdicio de alimentos. } \\
\text { - Potenciar el uso de materiales biode- } \\
\text { gradables, reciclables y reutilizables. } \\
\text { - Extender prácticas de ecoetiquetado. } \\
\text { - Gestionar de forma sostenible la } \\
\text { cadena de suministro. }\end{array}$ & $\begin{array}{l}\text { - Aprovisionamiento sostenible y } \\
\text { saludable } \\
\text { - Eficiencia productiva en ganaderías } \\
\text { - Modelo de producción eficiente } \\
\text { - Envases sostenibles } \\
\text { - Eficiencia ambiental } \\
\text { - Acciones e iniciativas internas y } \\
\text { externas para la prevención del } \\
\text { desperdicio de alimentos }\end{array}$ \\
\hline
\end{tabular}


'Pascual Aporta', un marco de gestión responsable para los objetivos de desarrollo sostenible

\begin{tabular}{|l|l|l|}
\hline & \multicolumn{1}{|c|}{$\begin{array}{c}\text { Retos identificados en el sector } \\
\text { alimentario }\end{array}$} & $\begin{array}{c}\text { Acciones de Pascual Aporta } \\
\text { alineadas con los ODS }\end{array}$ \\
\hline $\begin{array}{l}\text { ODS 13. } \\
\text { Acción por el } \\
\text { clima }\end{array}$ & $\begin{array}{l}\text { - Impulsar el cálculo y la reducción } \\
\text { de la huella ambiental. }\end{array}$ & $\begin{array}{l}\text { Programa de medio ambiente en } \\
\text { granjas proveedoras } \\
\text { - Modelo de producción eficiente } \\
\text { - Huella de carbono }\end{array}$ \\
\hline $\begin{array}{l}\text { ODS 15. } \\
\text { Vida de } \\
\text { ecosistemas } \\
\text { terrestres }\end{array}$ & $\begin{array}{l}\text { Impulsar la protección de los ecosis- } \\
\text { temas y la biodiversidad terrestre. }\end{array}$ & $\begin{array}{l}\text { Programa de medio ambiente en } \\
\text { granjas proveedoras } \\
\text { Aprovisionamiento de soja na- } \\
\text { cional de la máxima calidad y } \\
\text { seguridad }\end{array}$ \\
& $\begin{array}{l}\text { Protegemos y conservamos la } \\
\text { biodiversidad y los ecosistemas } \\
\text { de nuestro entorno }\end{array}$ \\
\hline
\end{tabular}

Por otro lado, Calidad Pascual, a través de su Dirección de Gestión Ética, Responsable y Excelente, forma parte de la Red Española del Pacto Mundial con la que colabora activamente en la difusión y sensibilización sobre los Objetivos de Desarrollo Sostenible.

La Red ha publicado en su web la Guía Sectorial ODS del sector agroalimentario en la que Calidad Pascual ha participado. En la misma se menciona el Plan de Movilidad Sostenible de la compañía, así como la iniciativa Pascual Saludable (actualmente convertida en el programa Vive tu Salud). Esta publicación destaca los retos y las oportunidades de negocio vinculadas a los Objetivos de Desarrollo Sostenible.

Asimismo, Calidad Pascual ha participado en el programa \#Companies4SDG que promueve desde el Pacto Mundial el conocimiento entre los empleados de las empresas de los Objetivos de Desarrollo Sostenible (ODS).

La Dirección de Gestión Ética, Responsable y Excelente ha organizado igualmente sesiones formativas con sus empleados de la mano de la Red Española del Pacto Mundial. Destaca más recientemente una sesión de formación, aprendizaje y sensibilización en torno a los Derechos Humanos y su impacto en la actividad de Pascual con la presencia de diversas áreas de la compañía. Mediante una jornada práctica, empleados estratégicos en Calidad Pascual han recibido una formación sobre los derechos humanos y se les ha dado las pautas necesarias para respetarlos en sus actividades diarias. En el taller se han tratado cuestiones como el consumo responsable, la seguridad de los clientes, las condiciones laborales de empleados y proveedores y la no discriminación desde un enfoque de derechos humanos. 